\title{
Kleinster gemeinsamer Nenner? Die Föderalismusreform II vor der Entscheidung
}

\author{
von Joachim Jens Hesse
}

Die „Föderalismusreform II“, gedacht als Fortsetzung der sich in ihrem ersten Teil auf Fragen der Kompetenzordnung richtenden ,Modernisierung der bundesstaatlichen Ordnung“, geht auf einen Einrichtungsbeschluss von Bundestag und Bundesrat vom 14. bzw. 15. Dezember 2006 zurück. Das Parlament nahm damit einen fraktionsübergreifenden Antrag von CDU/CSU, SPD und FDP gegen das Votum der Linken bei Enthaltung der Bündnisgrünen an; der Bundesrat fasste einen gleichlautenden Beschluss einstimmig. ${ }^{1}$ Die „Kommission zur Modernisierung der Bund-Länder-Finanzbeziehungen", so die präzise Bezeichnung des Gremiums, wurde dann am 8. März 2007 durch die Präsidenten von Bundestag und Bundesrat konstituiert, seitdem fungieren als Vorsitzende der SPDFraktionsvorsitzende im Bundestag, Peter Struck, sowie für den Bundesrat der Ministerpräsident Baden-Württembergs, Günther Oettinger. Die sich aus je 16 Mitgliedern von Bundestag und Bundesrat zusammensetzende Kommission kam bislang zu 13 Sitzungen zusammen, 115 Kommissionsdrucksachen zeugen von der geleisteten Arbeit. Die Kommission hat ihrem Titel gemäß den Auftrag, Vorschläge zur Modernisierung der Bund-Länder-Finanzbeziehungen zu erarbeiten, um diese den veränderten und für die Wachstums- und Beschäftigungspolitik wichtigen Rahmenbedingungen innerhalb und außerhalb Deutschlands anzupassen. Die Empfehlungen sollen dazu führen, die Eigenverantwortung der Gebietskörperschaften und deren aufgabengerechte Finanzausstattung zu stärken; eine den Einsetzungsbeschlüssen als Anlage beigefügte ,offene Themensammlung“ diente als Rahmen. Die Abhandlungen in diesem Sonderheft wenden sich vor allem den sog. „Verwaltungsthemen“ zu, ergänzt um zusammenfassende Erörterungen der Bund-Länder-Finanzbeziehungen. Ihnen ist - auf der Basis

1 Deutscher Bundestag, 16. Wahlperiode: Einsetzung einer gemeinsamen Kommission zur Modernisierung der Bund-Länder-Finanzbeziehungen, BT-Drs. 16/3885 vom 14.12. 2006 sowie Bundesrat: Einsetzung einer gemeinsamen Kommission zur Modernisierung der Bund-Länder-Finanzbeziehungen, BR-Drs. 913/06 (Beschluss) vom 15.12. 2006. 
einer ersten Sachverständigen-Anhörung ${ }^{2}$ - die Dokumentation in diesem Heft gewidmet.

\section{Erwartungen, Selbstverständnis und Ansatz der Kommissionsarbeiten}

Erwies sich schon die Zweiteilung der Bemühungen um eine Modernisierung der bundesstaatlichen Ordnung (Neugestaltung der Kompetenzordnung, Überprüfung der Bund-Länder-Finanzbeziehungen) aufgrund der politischen wie materiellen Komplexität als zwar verständlich, mit Blick auf die deutliche Interdependenz zwischen den beiden Teilen aber als fragwürdig, verwunderte auch die seitens der „Föderalismuskommission II“ vorgesehene Unterscheidung zwischen Finanzbeziehungen und Verwaltungsthemen. Natürlich ist einzuräumen, dass man damit glaubte, frühzeitigen Polarisierungen innerhalb eines ohnehin engen Zeitrahmens begegnen zu können, doch erwies sich der Versuch zur Arbeitsteilung bald als kontraproduktiv: Zu schnell „,verbiss“ man sich in Einzelfragen, fehlte der Bezug zur übergreifenden Fragestellung, stellten sich nicht zuletzt auch Abstimmungsprobleme mit den durch den ersten Teil der Föderalismusreform eingeführten Neuerungen.

Im Rahmen der konstituierenden Sitzung der Kommission sah man dies noch als Chance. So wies in seiner Einführung der Präsident des Deutschen Bundestages darauf hin, dass die Arbeit der Kommission zwar von hohen Erwartungen wie ausgeprägter Skepsis begleitet sei; gleichwohl konstatierte er eine gewisse Ablauflogik: Die Wege zu einer eigenverantwortlichen Aufgabenwahrnehmung von Bund und Ländern sollten von einer kritischen Analyse der öffentlichen Aufgaben und der Aufgabenstandards für die öffentliche Verwaltung begleitet sein, Vorbeugung und Bewältigung von Haushaltskrisen wären um einen Versuch zur Steigerung der Effizienz des staatlichen Handelns zu ergänzen. Die damit erneut angesprochene Zweiteilung geht auf die benannte Themensammlung zurück, die der Kommission gleichsam ab ovo und als „Relikt“ der „Föderalismusreform I“ übertragen wurde; ${ }^{3}$ zur Schlüsselfrage des Schuldenabbaus traten mithin Verwaltungs- und Organisationsfragen. Hier sollte dem Zusammenhang zwischen den

2 Sachverständigen-Anhörung zu den Finanzthemen vom 22.06. 2007.

3 Siehe hierzu die anlässlich der Verabschiedung der „Föderalismusreform I“ von Bundestag und Bundesrat gefassten Entschließungen: Deutscher Bundestag, 16. Wahlperiode: Entschließungsantrag der Fraktionen der CDU/CSU und SPD, BT-Drs. 16/2052 vom 28.06. 2006; Bundesrat: Beschluss des Bundesrates, BR-Drs. 462/06 vom 07.07. 2006. 
Aufgaben einerseits und der nötigen Finanzausstattung andererseits besondere Aufmerksamkeit gewidmet werden - und zwar länder- und ebenenübergreifend.

Berücksichtigt man darüber hinaus die „Tabuisierung“ von Kernbereichen (Finanzausgleich, Steuerneuverteilung, Länderneugliederung), ein Charakteristikum beider Kommissionen, verband sich damit trotz des politisch verständlichen und mit Blick auf potenzielle Polarisierungen und Blockaden vielleicht auch angezeigten Vorgehens der Verzicht auf ein Gesamtkonzept, das den Beratungen der Kommission „Rahmen und Programm“ hätte sein können. Punktuelle Reformen waren somit vorgezeichnet, trotz der Großen Koalition, von der man erwarten durfte, dass sie die Arbeiten der Kommission erleichtern, zumindest aber Mehrheiten einfacher machen würde. Dies freilich wurde schnell durch den Zeitfaktor begrenzt, nachdem der ,politische Terminkalender“ für die Beratungen und den Prozess der Konsensbildung in Bundestag wie Bundesrat nur bis Ende 2008 Zeit ließ.

\section{Verfahren: Selbstbeschränkung und Arbeitsteilung}

Nach der Anhörung von Sachverständigen zum Themenbereich „Finanzverfassung ${ }^{\text {“4 }}$ kam es am 8. November 2007 im Bundesrat zu einer erneut ganztägigen Anhörung, die sich jetzt auf die „Verwaltungsthemen“ konzentrierte. Sie wiederum baute auf einer Reihe sog. „Fachdiskurse“ auf, deren Ergebnisse später zusammengefasst wurden. Die dabei gewählte Ausdifferenzierung nach „Verwaltungskooperation, benchmarking, IT und Sonstiges" (Fachdiskurs 1), „Ausgabenautonomie, Standards - strukturell und sektoral -, Abweichungsrechte und Bandbreiten“ (Fachdiskurs 2) sowie „Steuerverwaltung, Verkehr und Sonstiges" (Fachdiskurs 3) dokumentiert ein arbeitsteiliges Verfahren. Die darin zum Ausdruck kommende Unterscheidung zwischen eher querschnittsorientierten Problemen, Standardfragen und sektoralen Politiken ist nachzuvollziehen, war aber von Beginn an unter den Vorbehalt zu stellen, dass die in ihnen zu erarbeitenden Ergebnisse wieder in einen Gesamtrahmen zurückgeführt werden müssten. Zum Zeitpunkt der Drucklegung dieses Heftes ist ein solcher Versuch bislang allerdings nicht erkennbar, verständlich aufgrund der Heterogenität der Fragestellungen, aber überprüfungsbedürftig angesichts der nicht beachteten wechselseitigen Bezüge.

4 Siehe hierzu die Dokumentation „Die ,Finanzthemen“ im Reformprozess: Kommissionsauftrag, Optionen, politische Diskussion“ in diesem Heft. 
Übersicht 1: Föderalstaatliche Organisationsentwicklung: Logik, Kriterien und Anforderungen

\section{Rahmenbedingungen und Anforderungen}

Laufender Bedarf (Status quo)

- Allgemeine Verwaltung (Finanzen, Personal, interner Service usw.)

- Justiz, Sicherheit, Außenbeziehungen

- Allgemeine und Sonderordnung (NaturUnwelt- und Verbraucherschutz usw.)

- Daseinsvorsorge (Straßen, Ver- und Entsorgung usw.)

- Soziales und Arbeit (aktive und passive Hilfe, Fürsorge usw.)

Kultur, Kultur und Wissenschaft (Schulen, Universitäten usw.)

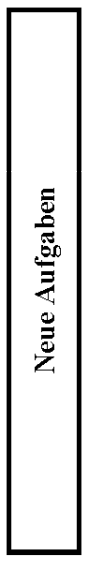

Exogene Anforderungen

- Entgrenzung (global/EU) $\rightarrow$ Wettbewerb und Regulation

- Veränderte Sicherheitslage $\rightarrow$ Regulation und Überwachung

- Klimawandel u. Ressourcenverknappung $\rightarrow$ Regulation,

Sicherungs- und Ordnungsmaßn.

- Technologischer Wandel $\rightarrow$ Regulation und Anpassung (e-Government)

\section{Endogene Anforderungen}

- Demographischer Wandel $\rightarrow$ Rückbau u. (Re-)Investitionen

- Soziale Frage $\rightarrow$,Hartz IV" usw.

- Alternde Gesellschaft, veränderte Erwerbsbiograph. $\rightarrow$ Bildungs-/Qualifizierungsbedarf

\section{Institutionelle Interessen}

- Eigenhoheit sowie Gestaltungs-/Steuerungsansprüche von Bund, Ländern und Kommunen

- Personalbelange, Beschäftigungsgarantien

Aufgaben- und Organisationsbestand
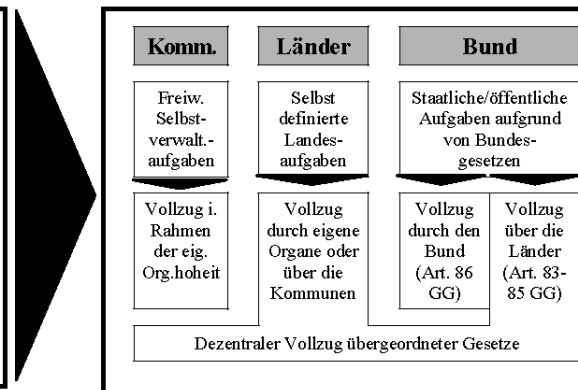

Dezentraler Vollzug übergeordneter Gesetze

\section{Wachstum durch neue und die} Intensivierung bestehender Aufgaben

(Folgen exogener Anforderungen)

$\begin{array}{ccc}\text { Kommunen } & \text { Länder } & \text { Bund } \\ \text { Komplexerer } & \text { Erweit. Auf- } & \text { Beständiger } \\ \text { Vollzug, } & \text { sichtsbezügc, } & \text { Bedarf an De- } \\ \text { Kapazitäts- } & \text { Koordinations-/ } & \text { und Re- } \\ \text { grenzen, } & \text { Kooperations- } & \text { regulierung, } \\ \begin{array}{c}\text { Investitions- } \\ \text { bedarf }\end{array} & \text { zwang } & \text { Voll- } \\ & & \text { zugsfragen }\end{array}$

(Folgen endogener Anforderungen)

Kommunen Länder Bund

Erw. Sozial- u. Förderbedarf, Finanzierungs-

Bildungsauftr., steigende Bil- und Förder-

Anpassung dungsausgaben, bedarf (Kitas

lokaler Infra- Aufsichtspraxis, SGB II usw.)

strukturen, IKZ Koordination

\begin{tabular}{|c|c|c|}
\hline Kommunen & Länder & Bund \\
Strukturerhalt, & Rückgewinn & Abbau v. Poli- \\
Forderung nach & autonomer & tikverflechtung, \\
frw./eigenstän- & Kern-/Gestal- & neue \\
dig gestaltbare & tungskom- & Steuerungsansp \\
Kompetenzen & petenzen & rüche \\
\hline
\end{tabular}




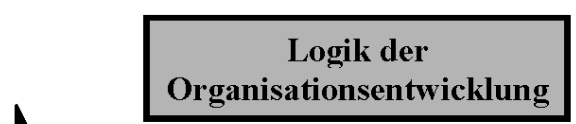

(1) Differenzierte Bestandserfassung Organisation

Staatsebene (Bd., Ld., Kommunen)

Un-/Mittelbare

Staatsverwaltung

- Vertikale

Differenzierung

(Instanzen)

- Rechts- u.

Strukturform

(Behörden,

Anstalten usw.)

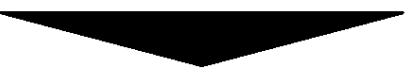

(2) Abschätzung v. Konsequenzen aus der Aufgabenentwicklung

- Europäischer Regulierungsdruck u. seine Auswirkungen (Ausschreibungen als Bsp.)

- Integrationsfähigkeit neuer Bedarfe in die ge gebene Organisation

- Notwendigkeit z. Schaff. neuer o

Erweiterung

bestehend.

Kapazität,

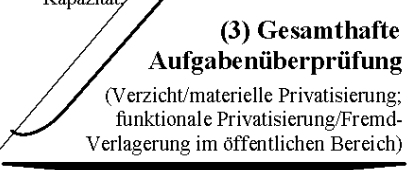

(4) Funktional-/Strukturreform

- Funktional: Zentralisierung,

Dezentralisierung, Verselbständigung

- Strukturell: Konzentration, Dislozierung

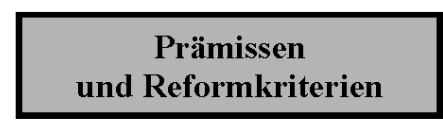

\section{Prämissen}

Beibehaltung des dreistufigen föderalen Systems

- Weitere Organisationsentwicklung im Rahmen der sowie ausgehend von der Föderalismusreform

- Beurteilung der Eignung von Reformoptionen aus gesantstaatlich er Sicht und nicht nur aus der Perspektive einer gebietskörperschaftlichen Ebene (Auseinan dersetzungen um Schulträgerschaften, SGB II und die Steuerverwaltung als Beispiele)

Entflechtung, Stärkung eigenständiger Gestaltungs- u. Regulierungskompetenzen

- Verstärkte ebenenübergreifende

Koordination in Querschnittsbereichen (IT. Organisationsstan dards, Kostenvergleiche usw.) sowie bei essentiellen Ordnungs- und Sicherheitsfragen

- Erfordernis einer verstetigten

Organisationsentwicklung auf der Basis nationaler wie internationaler Vergleiche

\begin{tabular}{l}
\hline Operative Reformkriterien \\
\hline - Effizienz: Wirtschaftlichkeit und \\
Zielgenauigkeit, flexible und \\
bedarfsgerechte Ressourcensteuerung \\
- Effektivität: Steuerungsfähigkeit, Qualität, \\
Zielgenauigkeit, Reaktionsvermögen \\
- Bürgernähe und Teilhabe: Legitimation, \\
Kontrolle, Repräsentativität, Zugänge \\
- Politisch-administrative Durchsetzbar- \\
keit: Akzeptanz durch internc (Pcrsonal, \\
Amtsträger) u. externe Akteure (Offtl., \\
Parteien)
\end{tabular}

\section{Quelle: Eigene Darstellung.}


Die vorstehende Übersicht 1 macht die sich damit verbindenden Schwierigkeiten und Probleme deutlich. Sie verweist in einem funktionsanalytischen Zugriff auf zahlreiche Verflechtungen, die bei der Bearbeitung von Einzelfragestellungen Berücksichtigung finden müssten; zudem dokumentiert sie den hochpolitischen Charakter eher formaler Analyseansätze. Die Darstellung beruht nicht nur auf gleichsam logischen Interdependenzen im Rahmen einer Föderalismusreform, sondern auch und gerade dem Einblick in laufende Reformprozesse auf Bundes-, Landes- und kommunaler Ebene. ${ }^{5}$ Sie verweisen jenseits der Schwierigkeit, „einfache“ Lösungen zu identifizieren, auf den in kooperativ angelegten föderalstaatlichen Systemen hohen Koordinationsbedarf - und zwar horizontal wie vertikal. Während horizontal etwa das Verhältnis zwischen den Akteuren (oder Ressorts) auf der jeweiligen Ebene von Bedeutung ist (und insbesondere bundesseitig gröblich unterschätzt wird), stellen die vertikalen Abstimmungs- und Koordinationsleistungen den eigentlichen Schwerpunkt des hier zur Diskussion stehenden Reformansatzes dar. Allerdings lassen nur wenige der im Rahmen der „Föderalismuskommission II“ vorgelegten Papiere erkennen, dass man sich dieser Problematik bewusst ist, so dass entsprechende Versäumnisse schnell zu Polarisierungen zwischen den Beteiligten führen können. Der Ausweis einer solchen Sachlogik des Reformprozesses erscheint mithin angezeigt, nicht primär als Ausfluss eines systematisierenden wissenschaftlichen Interesses, sondern unter Berücksichtigung der politischen „Machbarkeit“ einzelner Reformvorstellungen.

Ein weiteres Kriterium zur Beurteilung der laufenden Arbeiten der „Föderalismuskommission II" kann dadurch gewonnen werden, dass man zwischen dem normativen und dem funktionalen Gehalt der Erörterungen unterscheidet. Wer die Protokolle der Kommissionssitzungen einsieht und die „Fachdiskurse“ nachvollzieht, wird durchgängig mit einer gewissen Verkürzung der Diskussion konfrontiert: dem verständlichen Versuch, durch die schnelle Vorlage eines generalisierenden Reformansatzes die Agenda zu dominieren, sowie den meist weniger „wirksamen“ Bemühungen, unter Verweis auf die Umsetzbarkeit entsprechender

5 Unter den jüngeren Arbeiten des Autors s. vor allem Hesse, J.J.: Aufgabenkritik, Funktional- und Strukturreform in den Flächenländern, Baden-Baden, 2007; ders.: Verwaltungsstruktur- und Funktionalreform in Schleswig-Holstein, Berlin, 2007; ders.: Raumordnung und Landesentwicklung. Reformoptionen für ein tradiertes Politikfeld, Baden-Baden, 2006; ders.: Neubau von Metropolverwaltungen, Baden-Baden, 2008, i.E.; ders./Götz, A.: Kooperation statt Fusion? Interkommunale Zusammenarbeit in den Flächenländern, Baden-Baden, 2006; Hesse, J.J./Götz, A./Schubert, S.: Reform der Hoheitsverwaltung, Baden-Baden, 2007. 
Vorstellungen gleichsam $a b$ ovo konsensbildend wirken zu wollen. Ob Steuerverwaltung, IT-Struktur oder benchmarking, es findet sich meist eine immer wiederkehrende Argumentation: der Vortrag eines ja oder nein, ohne den besonderen funktionalen Voraussetzungen, Erwartungen und Interessen gerecht zu werden. Dadurch „erledigte“ sich manche Reformvorstellung gleichsam von selbst, kam es in anderen (das benchmarking als Beispiel) zu realitätsfernen Erwägungen, die erkennbar ohne Berücksichtigung der methodischen und funktionalen Voraussetzungen eines derart anspruchsvollen Instrumentariums formuliert wurden. Für die Entscheidungsphase dieser wie künftiger Reformkommission(en) bleibt es angeraten, die in den Einsetzungsbeschlüssen meist zu Recht benannte „Aufgabenkritik“ tatsächlich auch zu leisten, also der funktionalen Ausgangssituation zu entsprechen, bevor normativ geprägte Vorstellungen oder gar Entwürfe für Verfassungsänderungen vorgetragen werden. Ein kontinuierlicher Nachvollzug der Aufgabenwahrnehmung und ihres nicht nur rechtlichen, sondern vor allem auch organisatorischen und funktionalen Rahmens ist unabdingbar, nimmt man das Ziel einer „Modernisierung der bundesstaatlichen Ordnung" ernst.

\section{Die Finanzverfassung: die Verschuldungsfrage im do ut des}

Nach der bereits frühzeitig erfolgten Einschränkung der finanzverfassungsrelevanten Fragestellungen auf die Verschuldungsproblematik zeichnet sich zum Zeitpunkt des Erscheinens dieses Heftes ab, dass sich die Föderalismuskommission auf das Konzept einer sog. „Schuldenbremse“ einigen könnte. So wird erkennbar, dass es insbesondere den Sozialdemokraten gelang, die in Teilen sehr unterschiedlichen Vorstellungen zu harmonisieren und die Positionen des Bundesfinanzministers, des SPD-Fraktionsvorsitzenden und (Ko-)Vorsitzenden der Föderalismuskommission sowie einzelner Ländervertreter aufeinander zu beziehen. Danach soll sich der Bund in konjunkturellen „Normalzeiten“ noch mit $0,5 \%$ des Bruttoinlandsprodukts verschulden dürfen. Während bei einem Aufschwung die Nettokreditaufnahme weniger als $0,5 \%$ betragen sollte, wären bei einem konjunkturellen Abschwung bis zu $3 \%$ erlaubt; die Länder dürften zudem weitere $0,25 \%$ des BIP an Schulden aufnehmen. In Fällen, in denen die Regierung für unerwartete Mehrausgaben oder Mindereinnahmen nicht selbst verantwortlich ist, bliebe nach diesen Vorstellungen eine höhere Verschuldung zugelassen. Benannt werden als Beispiele mögliche Finanzmarktkrisen und ein plötzlich ausbleibender Bundesbankgewinn. Gleiches gälte für Ausnahmesituationen wie Naturkatastrophen oder Terroranschläge. Strittig bleibt, wer über eine 
solche „Ausnahmesituation“ entscheidet. Die Positionen sehen hier entweder den Bundestag mit seiner Kanzlermehrheit oder aber zwei Drittel aller Stimmen im Parlament vor. Schließlich wird erörtert, in nachhaltigen wirtschaftlichen Abschwungphasen kreditfinanzierte Ausgaben möglich zu machen, um handlungsfähig zu bleiben.

Mehrausgaben oder Mindereinnahmen, also etwa Steuersenkungen ohne Gegenfinanzierung, wären nach diesen Vorstellungen nur noch dann erlaubt, wenn im Bundestag eine deutlich größere Mehrheit zustimmt, als sie bislang erforderlich ist. Allerdings verbleibt unklar, wie diese unbestimmten Begrifflichkeiten in der Praxis gefüllt werden sollen, ob etwa Steuergesetze nur noch mit einer Zweidrittelmehrheit erlassen werden könnten oder welche anderen Abstimmungs- und Mehrheitsregeln Platz greifen sollten. Konsens herrscht lediglich dahingehend, dass ein Missbrauch von Ausnahmetatbeständen durch ein sog. „Kontrollkonto“ zu verhindern ist, auf dem im Jahresverlauf Fehlentwicklungen als Last- und Mehrerlöse als Gutschrift gebucht werden sollten. Freilich ist ungeklärt, ob es bei der Führung eines solchen „Berichtskontos“ bleibt (in der Hoffnung, dass eine entsprechende Veröffentlichung mit nachfolgenden Konsequenzen verbunden ist) oder ob nicht doch Bund wie Länder dazu gezwungen werden sollten, beim Überschreiten eines festzulegenden Fehlbetrags zusätzliche Einsparungen vorzunehmen. Nach vorliegenden Berechnungen würde in Verfolgung dieses Konzeptes der Schuldenstand Deutschlands, der derzeit bei etwa 1,6 Bio. Euro liegt, weiter steigen, da in konjunkturell normalen oder auch guten Zeiten Defizite erlaubt wären, doch würde die Verschuldung langsamer zunehmen als die gesamtstaatliche Wirtschaftsleistung. Die Schuldenquote, also das Verhältnis des Schuldenstandes zum BIP, nähme langsam ab.

Folgt man den bislang vorliegenden Vorstellungen zu dieser Frage, ${ }^{6}$ böte sich so in der Tat eine beide Seiten der Großen Koalition befriedende Perspektive, der Schuldenentwicklung der Gebietskörperschaften nicht länger tatenlos gegenüber zu stehen, sondern ihr wenigstens einen Rahmen zu geben, zumal nach der Ausblendung des Finanzausgleichs und aufgrund einer voraussichtlich nicht konsensfähigen Steuerneuverteilung die Kommission dringend eines Erfolges in dieser Schlüsselfrage der bundesstaatlichen Entwicklung bedarf. Dies freilich als „Reform der bundesstaatlichen Finanzverfassung“ zu bezeichnen, sollte überdacht werden: zu offensichtlich das Bemühen, wenigstens in einer Teilfrage Konsens zu erzielen, unterschiedlichste Positionen einzubeziehen und zu erken- 
nen zu geben, dass die viel zitierte Generationengerechtigkeit in den Beratungen der Föderalismuskommission ernst genommen wurde. Eine Strukturreform der Finanzverfassung verbindet sich damit nicht.

\section{Verwaltungsthemen: der ungeliebte Appendix}

Die von der Kommission als wichtig erachteten Verwaltungsthemen kamen einer laundry list gleich. Darin dokumentierte sich nicht nur ein spezifischer „Reformstau“, sondern auch ein Informations- und Transparenzdefizit zwischen den Beteiligten. Dies erklärt sich zum einen aus den im Verwaltungsbereich besonders deutlichen Abstimmungs- und Koordinationsmängeln, zum anderen aus der bis heute erstaunlichen Asymmetrie der gebietskörperschaftlichen Reformbereitschaft. Während die Gemeinden bereits frühzeitig aufgrund der hier besonders drängenden Haushaltsprobleme zu vergleichsweise weitgehenden Strukturreformen fanden und substantielle Ansätze zu einer Aufgabenkritik verfolgten, zogen die Länder mit zeitlicher Verzögerung, ab Mitte der 1990er Jahre aber in großer Intensität nach. Ähnliches findet sich auf der Bundesebene nicht. Hier ist der Großen Koalition lediglich zuzugestehen, dass mit einer großflächigen Standardkostenkontrolle Nachholbedürfnissen entsprochen wird. Allerdings ist darin bislang nicht mehr als eine fast selbstverständliche formale Entbürokratisierung zu sehen. Fehlender politischer Wille, Ressortegoismen und ein gelegentlich durchaus arrogantes Verhalten den anderen Gebietskörperschaften gegenüber mögen dieses Defizit erklären, das die Bundesrepublik im Rahmen der Verwaltungspolitik größerer EU-Mitgliedstaaten zu einem „Nachzügler“ macht. Insofern ist es zu begrüßen, dass die im Fragenkatalog der Kommission zutage tretende „Kritikwürdigkeit“ jetzt auch nach außen hin dokumentiert, wie groß der Reformbedarf ist und welche Fragen in besonderer Weise hiervon betroffen sind.

Blickt man auf die einzelnen Themenschwerpunkte, wird freilich auch hier deutlich, dass die erwartbaren Ergebnisse von den eingangs benannten Selektivitäten und Filtern gekennzeichnet sind. Von einer den Namen verdienenden Aufgabenkritik kann nicht oder bestenfalls in Ansätzen gesprochen werden, nimmt man etwa Schlüsselfragen, wie die der Steuer- und der Arbeitsverwaltung, als Beispiel. $^{7}$ Beide Politikfelder weisen in großer Dringlichkeit auf eine fraglos suboptimale Verwaltungsstruktur hin, ohne dass bislang Lösungsansätze erkennbar

7 Vgl. die Anlagen A-4 und A-5 in: Hesse, J.J.: Stellungnahme im Rahmen der Sachverständigen-Anhörung der Kommission von Bundestag und Bundesrat zur Modernisierung der Bund-Länder-Finanzbeziehungen - Verwaltungsthemen -, K-Drs. 078 vom 24. 10. 2007, $81 \mathrm{ff} ., 104 \mathrm{ff}$. 
wurden. Während in der Steuerverwaltung die Bund-Länder-Divergenzen hinsichtlich des Steuerungsanspruches und einer etwaigen Zentralisierung in besonderer Weise zutage treten, stellt die Arbeitsverwaltung seit dem Urteil der Bundesverfassungsgerichts zur Frage der Aufgabenträgerschaft nach dem SGB II ${ }^{8}$ ein Schlüsselproblem für die Kommission dar. Der Autor hat sich hierzu vielfach, auch vor dem Bundesverfassungsgericht ${ }^{9}$, geäußert, so dass an dieser Stelle eine nähere Darstellung entfallen kann. Allerdings sei darauf verwiesen, dass für dieses Aufgabenfeld inzwischen Lösungsansätze vorliegen, die auch und gerade für die Föderalismuskommission von Bedeutung sein sollten. ${ }^{10}$

Die Diskussion um ein verpflichtendes benchmarking schließlich macht erneut und exemplarisch deutlich, wie schwierig es ist, föderalstaatliche Querschnittsfragen konsensfähig zu machen, erst recht, wenn sie auf eine erweiterte Transparenz und wechselseitige Information zielen. Im Hintergrund steht die beklagenswert geringe Kooperation zwischen den Gebietskörperschaften samt der sich mit polarisierten Abstimmungsverfahren verbindenden Zeit-, Ressourcen- und Steuerungsverluste. Ziel ist deshalb die Durchführung von Leistungsvergleichen, um Verwaltungsverfahren und deren Ergebnisse zu verbessern.

Bei diesen Fragen wird einmal mehr erkennbar, wie wenig funktional basiert die Diskussion bislang geführt wurde. So schlägt der Bund eine Institutionalisierung des benchmarking durch die Einfügung eines neuen Abschnitts in Art. 91 GG vor, allerdings meist ohne empirische Begründung und ohne einen erkennbaren Nachvollzug der schwierigen methodischen Voraussetzungen. Hinzu kommt, dass sich der Bund selbst von allen Bemühungen um eine Ressortabstimmung und ein horizontales benchmarking ausnimmt, seine Rolle als Promotor der Idee mithin selbst in Frage stellt. Wird dies zudem mit fragwürdigen Institutionalisierungsansätzen (einer benchmarking-Agentur) verbunden und den Ländern gleichsam in der Form eines Diktums begegnet, ist ein Scheitern vorgezeichnet. So wird verständlich, dass derzeit ,weichere“, aber seitens der beteiligten Akteure akzeptablere Alternativen diskutiert werden, etwa die Einführung einer „Strukturberichterstattung für die deutschen Gebietskörperschaften““11. Dieser

8 BVerfG, Urt. v. 20. 12. 2007, 2 BvR 2433/04, 2 BvR 2434/04.

9 Hesse, J.J.: Circulus vitiosus: Die Mischverwaltung nach dem SGB II als organisations- und leistungspolitisches Dilemma, in: Hesse, J.J./Götz, A.: Für eine zukunftsfähige Arbeits- und Sozialverwaltung. Aufgabenträgerschaft nach dem SGB II („Hartz IV“) 2005 - 2007, Baden-Baden, 2007, 209-224.

10 Hesse, J. J.: Hartz IV als Chance, in: FAZ vom 04. 06. 2008, 10.

11 Hesse, J.J.: Der Bund in der Verantwortung: Plädoyer für eine nachhaltige Modernisierung von Regierung und Verwaltung, in: ZSE, 5/1 (2007), 99-111; ders.: Stellungnahme, a. a. O., $113 \mathrm{ff}$. 
Ansatz erfährt große Unterstützung, weil er zunächst darauf setzt, die Informationsbasis der Beteiligten nachhaltig zu verbessern und erst auf dieser Grundlage die Möglichkeit zu schaffen, über eine späterhin erweiterte Transparenz punktuelle Vergleiche zuzulassen. Nur so dürfte es angesichts der weit ausdifferenzierten Verwaltungslandschaft Deutschlands möglich sein, zu einer Leistungsüberprüfung und nachfolgenden Verbesserung des Verwaltungshandelns beizutragen. Dass diese wiederum nur als Prozess zu gestalten sind, sollte angesichts des nationalen wie internationalen Diskussionsstandes selbstverständlich sein.

\section{Durchbruch zur Reform oder punktuelle Anpassung?}

Dieses Themenheft der ZSE erscheint zeitgleich mit der Vorlage sog. „Eckpunkte" durch die beiden Kommissionsvorsitzenden. Natürlich sind dabei Überraschungen nicht ausgeschlossen, mit Blick auf die Reichweite der Reformvorstellungen aber auch nicht wirklich zu erwarten. Die ursprünglich als besonders günstig eingeschätzten Rahmenbedingungen der Großen Koalition haben sich verschlechtert, die Nähe zum Wahljahr 2009 macht größere Eingriffe, mithin Strukturreformen, unwahrscheinlich. Im Ergebnis dürfte es deshalb, trotz sich schnell verändernder Rahmenbedingungen, zu einer nur punktuellen Anpassung des deutschen Föderalismus kommen. Die nachfolgenden Beiträge nehmen dies auf und versuchen in einem Kreis sachkundiger, d.h. der Empirie des Föderalismus und des politisch-administrativen Handelns zugewandter Wissenschaftler, Lösungsansätze zu präsentieren. Sie sind als Erinnerung und Anregung zu verstehen, gelegentlich auch als Mahnung, die Chancen der Föderalismusreform nicht durch die Verfolgung administrativer Routinen und ausschließlich parteipolitisch geprägte Prioritäten einzuschränken. 